\title{
Weighted stability number of graphs and weighted satisfiability: The two facets of pseudo-Boolean optimization
}

\author{
D. de Werra · P. L. Hammer
}

Published online: 2 December 2006

(C) Springer Science + Business Media, LLC 2007

\section{Foreword}

In the break following the first hour of a Boolean seminar held in the late '70s at the Ecole Polytechnique Fédérale de Lausanne, one of the participants approached us with a fascinating idea. He told us that he had noticed that by associating to a graph a certain pseudo-Boolean function, simplifying afterwards a particular posiform of that function, and finally associating a graph to the resulting expression, the stability number of the original graph would be reduced by exactly one unit in this sequence of transformations.

The name of the seminar participant who made this remark was Christian Ebenegger, an economist by training, a member of the Faculty of Architecture, and an enthusiastic believer in operations research. Following Christian's weekly persistent pointers to more and more convincing examples of graphs whose stability number he had calculated by repeated applications of the above transformations, it was finally realized that his proposed transformations were not only valid, but did in fact always yield the desired result.

Christian left us suddenly and prematurely in 2002. It is to his memory that we dedicate this note.

D. de Werra $(\bowtie)$

IMA - EPFL, Lausanne, Suisse

e-mail: dewerra.ima@epfl.ch

P. L. Hammer

RUTCOR, Rutgers University, 640 Bartholomew Rd, Piscataway NJ 088254-8003, USA

e-mail: hammer@ rutcor.rutgers.edu 


\section{An introduction}

Among the integer programming problems that attracted the attention of many researchers, the maximum weight stable set problem (MWSP) has generated a variety of contributions based on very different techniques.

Given a graph $G=(V, E)$ where each vertex $i$ has a (positive) weight $w(i)$ one wants to find a stable set $S$ (i.e., a subset $S \subseteq V$ of vertices such that no two vertices in $S$ are linked by an edge (Berge, 1973) with maximum weight $w(S)=\sum(w(i) \mid i \in S)$. It is formulated as follows:

$$
\begin{array}{lll}
\operatorname{Max} \mathrm{z}= & \sum_{i=1}^{n} w(i) x_{i} \equiv \alpha_{w}(G) & \\
\text { s.t. } & x_{i}+x_{j} \leq 1 & \text { for all }[i, j] \in E \text { (MWSP) } \\
& x_{i} \in\{0,1\} & \text { for all } i \in V
\end{array}
$$

When all weights $w(i)$ are one $\alpha_{w}(G)$ will be written $\alpha(G)$; it is the stability number of $G$.

\section{A formulation in pseudo-Boolean terms}

As observed by Hamor (1980), this problem can also be viewed as the maximization of a pseudo-Boolean function, i.e., a real-valued function of Boolean variables. It is obtained as follows: we take a family of complete bipartite graphs (not necessarily induced) $G_{j}=$ $\left(A_{j}^{*} ; B_{j}^{*} ; E_{j}\right)(j=1, \ldots, q)$ that cover the edge set $E$, we associate to each $G_{j}$ a 0 - 1 variable $x_{j}$, and we set:

$$
f\left(x_{1}, \ldots, x_{n}\right)=\sum_{i \in V} w(i) T_{i}
$$

where

$$
T_{i}=\prod_{j \in A_{i}} x_{j} \prod_{k \in B_{i}} \bar{x}_{k}
$$

and

$$
A_{i}=\left\{j: i \in A_{j}^{*}\right\}, B_{i}=\left\{j: i \in B_{j}^{*}\right\}
$$

Notice that this representation of $f$ is a posiform (there is no constant term and all coefficients $w(i)$ are positive). Clearly the maximum of $f$ will give the maximum weight of a stable set in $G$ : each $T_{i}$ with value 1 will correspond to a vertex $i$ of $G$ which will be included in the set $S$; it follows from the construction of $f$ that no two adjacent vertices of $G$ can both be in $S$.

In fact given any pseudo-Boolean function $g$, we can express it as $g=K+f$ where $K$ is a constant and $f$ a posiform, by using the equality $\bar{x}_{j}=1-x_{j}$.

Any given pseudo-Boolean function $g$ can generally be written as a constant plus a posiform in many different ways. 


\section{The conflict graph and its use}

Maximizing $g$ amounts to maximizing an associated posiform $f$; it was also observed that conversely maximizing a posiform $f$ can be reduced to finding a stable set with maximum weight in a graph $G_{f}=(V, E)$ called the conflict graph of $f$, and defined in the following way:

If $f=\sum_{i=1}^{n} w(i) T_{i}$ where each $T_{i}$ is defined by (1) where $A_{i}, B_{i} \subseteq\{1, \ldots, n\} A_{i} \cap B_{i}=$ $\emptyset$, then to each $i$ we associate a vertex $i$ of $G_{f}$ with weight $w(i)$, and define the edge set by $E=\left\{[i, j]:\left(A_{i} \cap B_{j}\right) \cup\left(A_{j} \cap B_{i}\right) \neq \emptyset\right\}$. This means that in $G_{f}$ there is an edge between vertices $i$ and $j$ if the terms $T_{i}$ and $T_{j}$ are in conflict (there is a $k$ such that $x_{k}$ occurs in one term and $\bar{x}_{k}$ in the other term).

It is clear that $\max \left\{w(S): S\right.$ stable set in $\left.G_{f}\right\}=\max f\left(x_{1}, \ldots, x_{n}\right)$.

So the problem of finding a stable set of maximum weight in a graph $G$ is equivalent to finding the maximum of a pseudo-Boolean function (and in particular of a posiform).

\section{The struction}

Now if we have a graph $G=(V, E)$ with positive weights on its vertices, there are many different ways of covering its edges by complete bipartite graphs; as a consequence there are many different posiforms that could be maximized to find $\alpha_{w}(G)$.

Since there is usually a choice, one may wonder whether there are some "good" choices to be made. This is precisely what Christian Ebenegger noticed and this idea was exploited in Ebenegger, Hammer, and de Werra (1984) for defining the struction (from STability number RedUCTION).

For any vertex $v$ of a graph, let $N(v)$ be the set of its neighbors. Consider now a graph $G=$ $(V, E)$ and choose a vertex $a_{o}$ in $V$; Assume $N\left(a_{o}\right)=\left\{a_{1}, \ldots, a_{p}\right\}$ and let $R=V-N\left[a_{o}\right]=$ $\left\{a_{p+1}, \ldots, a_{|V|-1}\right\}$ where $N\left[a_{o}\right]=N\left(a_{o}\right) \cup\left\{a_{o}\right\}$. We construct the following covering of the edge set $E$ by $|V|-1$ stars:

(a) For each $i=1, \ldots, p$, we take the star with vertex set

$$
\left\{a_{i}, a_{o}\right\} \cup\left\{a_{j} \in N\left(a_{i}\right) \cap N\left(a_{o}\right): j>i\right\} \cup\left(N\left(a_{i}\right) \cap R\right)
$$

and with center $a_{i}$.

(b) For each $i=p+1, \ldots,|V|-1$, we take the star with vertex set

$$
\left\{a_{i}\right\} \cup\left\{a_{j} \in N\left(a_{i}\right) \cap R: j>i\right\}
$$

and with center $a_{i}$.

One obtains the posiform

$$
f=\sum_{i=o}^{|V|-1} w\left(a_{i}\right) T\left(a_{i}\right)
$$

where

$$
T\left(a_{o}\right)=\prod_{i=1}^{p} \bar{x}_{i}
$$




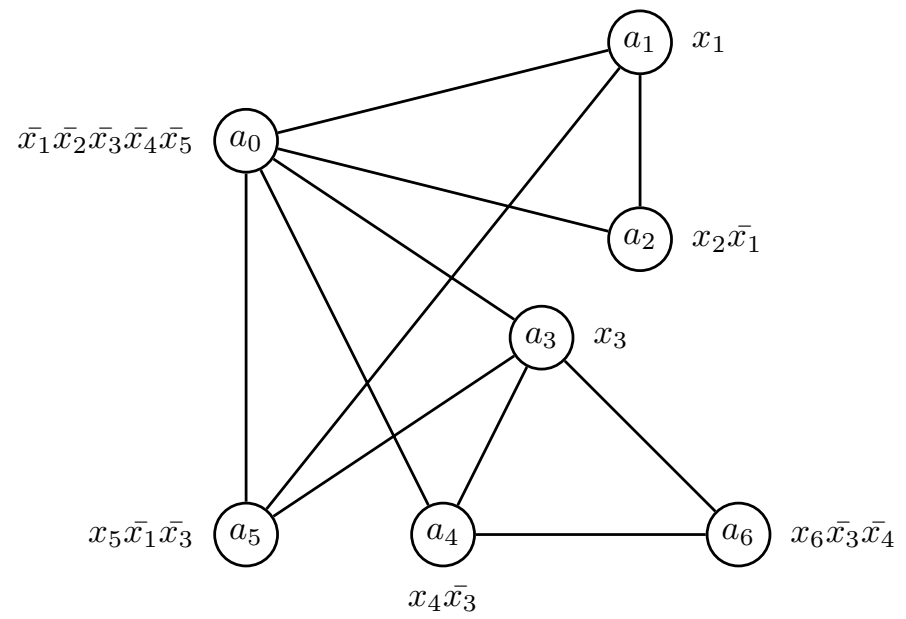

Fig. 1 A graph $G$ with the posiform $f$

$$
T\left(a_{i}\right)=\left\{\begin{array}{l}
x_{i} \prod_{\substack{a_{j} \in N\left(a_{i}\right) \\
1 \leq j<i \leq p}} \bar{x}_{j} \quad(1 \leq i \leq p), \\
x_{i} \prod_{a_{j} \in N\left(a_{i}\right) \cap N\left(a_{0}\right)} \bar{x}_{j} \prod_{\substack{a_{j} \in N\left(a_{i}\right) \cap R \\
p<j<i<|V|}} \bar{x}_{j}
\end{array}\right.
$$

For simplicity purposes we shall assume that all weights are one. An example is given in Fig. 1. For this example, after the transformation of $f$, we get

$$
f=1+x_{1} x_{3}+\bar{x}_{1} x_{2} x_{3}+\bar{x}_{3} x_{1} x_{4}+\bar{x}_{1} \bar{x}_{3} x_{2} x_{4}+\bar{x}_{1} \bar{x}_{3} x_{2} x_{5}+\bar{x}_{1} \bar{x}_{2} \bar{x}_{3} x_{4} x_{5}
$$

In general, this covering is always such that $\sum_{i=o}^{|V|-1} T\left(a_{i}\right)$ can be rewritten as:

$$
1+\sum_{\substack{a_{q} \notin N\left(a_{r}\right) \\ 1 \leq q<r \leq p}} x_{q} x_{r} \prod_{1 \leq s<q} \bar{x}_{s} \prod_{\substack{a_{t} \in N\left(a_{r}\right) \\ q<t<r}} \bar{x}_{t} .
$$

which is of the form $1+g$, where $g$ is a posiform. So in case all weights $w\left(a_{i}\right)$ are one, we have $f=1+g$. Since $g$ is a posiform, we can now consider its conflict graph, which we call $G\left(a_{o}\right)$; for the example of Fig. 1, we get the graph given in Fig. 2.

Since $\alpha(G)=\max f=1+\max g$, for the graph $G\left(a_{o}\right)$, we have $\alpha\left(G\left(a_{o}\right)\right)=\alpha(G)-1$.

\section{A direct reduction}

In fact $G\left(a_{o}\right)$ could have been obtained directly from $G$ by the following transformation:

(1) remove vertices $a_{o}, a_{1}, \ldots, a_{p}$ from $G$

(2) add to the rest $R$ of the graph a set of new vertices

$$
W=\left\{v_{i j}: 1 \leq i<j \leq p \text { and }\left[v_{i}, v_{j}\right] \notin E\right\}
$$


Fig. 2 The graph $G\left(a_{o}\right)$ for the graph $\mathrm{G}$ of Fig. 1

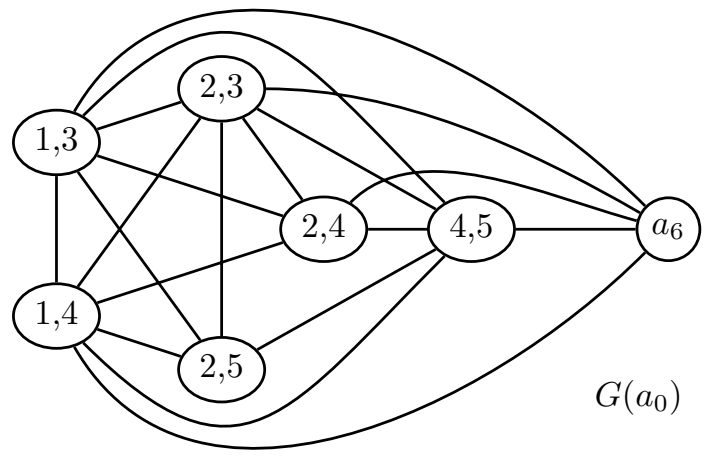

(3) join two new vertices $v_{i, j}$ and $v_{k, l}$ by an edge whenever $i \neq k$ or if $\left[v_{j}, v_{\ell}\right] \in E$; join a new vertex $v_{i, j}$ to a vertex $u \in R$ by an edge whenever $\left[v_{i}, u\right] \in E$ or $\left[v_{j}, u\right] \in E$.

One can then show that $\alpha\left(G\left(a_{o}\right)\right)=\alpha(G)-1$; but it is worth insisting on the fact that this construction was inspired by pseudo-Boolean manipulations and the choice of an appropriate covering of edges of $E$ by complete bipartite graphs was crucial for deriving this reduction of the stability number $\alpha(G)$.

\section{Using the struction in an algorithm}

We may now think of computing $\alpha(G)$ by repeatedly applying the struction until one gets a graph $G^{k-1}$ with $\alpha\left(G^{(k-1)}\right)=1 ; G^{(k-1)}$ is a clique and we get $\alpha\left(G^{(o)}\right)=\alpha(G)=k$.

This does clearly not provide a polynomial algorithm for a general graph $G$; the number of vertices in the consecutive graphs $G^{(\ell)}$ may increase exponentially.

However in special classes of graphs, by making systematically good choices of the center $a_{o}$ of the struction, one may avoid this inconvenience; this was exploited for some subclasses of claw-free graphs for instance (see Hammer, Mahadev, and de Werra, 1985).

For a weighted graph $G$, the basic struction operation can be extended to reduce $\alpha_{w}(G)$ by a fixed amount (for instance, the minimum weight of the nodes of $G$ ), but we shall not discuss this here.

The example of the struction is an interesting illustration of a situation in which pseudoBoolean techniques have led to the discovery of some graph transformation (reducing the stability number in our case) which could afterwards be explained and justified directly. In that sense pseudo-Boolean algebra has played a role of catalyst in the procedure of designing a graph transformation.

There are other situations where the same phenomenon occurred (see for instance (Alexe et al., 2004; Hertz, 1995, 1997) for variations and extensions of such transformations).

\section{Another reduction of pseudo-Boolean inspiration}

It is worth mentioning also the case of "magnets" that generalize a simple neighborhood reduction. A magnet in a graph $G=(V, E)$ is a pair $(a, b)$ of adjacent vertices such that every vertex in $N(a)-N(b)$ is adjacent to every vertex in $N(b)-N(a)$. If $(a, b)$ is a magnet 

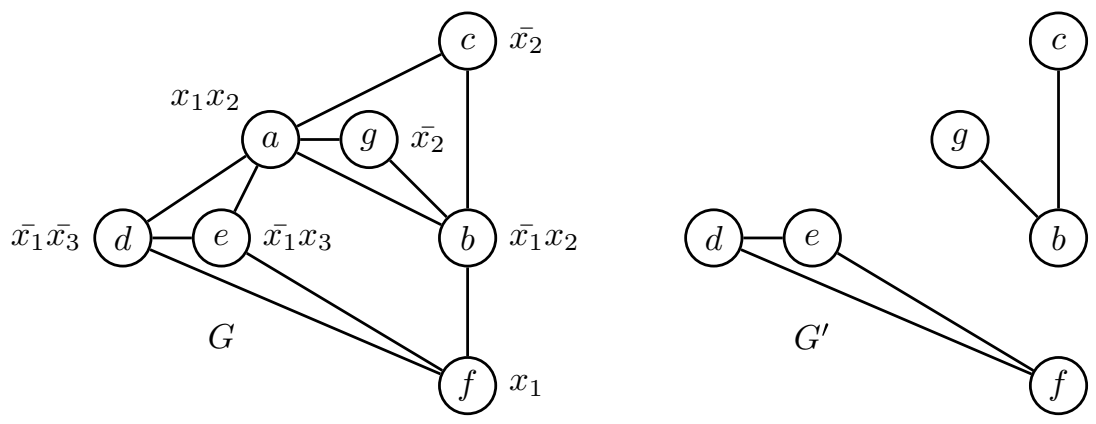

Fig. 3 The magnet transformation

in $G$, then the edges adjacent to $a$ or $b$ can be covered by the following two complete bipartite subgraphs $G_{1}=\left(N(b)-N(a), N(a)-N(b), E_{1}\right)$ and $G_{2}=\left(\{a, b\}, N(a) \cap N(b), E_{2}\right)$ of $G$. If we cover the edges of $E-\left(E_{1} \cup E_{2}\right)$ with complete bipartite subgraphs $G_{3}, \ldots, G_{q}$, then the associated posiform $f=\sum_{v \in V} T(v)$ is such that $T_{a}=x_{1} \cdot x_{2}, T_{b}=\bar{x}_{1} \cdot x_{2}$, so we have $T_{a}+T_{b}=\left(x_{1}+\bar{x}_{1}\right) x_{2}=x_{2}$ (assuming all weights equal to one). Hence $f$ has the same maximum as $g=\sum_{v \in V-\{a, b\}} T(v)+x_{2}$.

The conflict graph $G^{\prime}=\left(V^{\prime}, E^{\prime}\right)$ associated with $g$ satisfies $\left|V^{\prime}\right|=|V|-1$ and $\alpha\left(G^{\prime}\right)=$ $\alpha(G)$. This construction is illustrated in Fig. 3 .

Again, one could have constructed directly the graph $G^{\prime}$ by deleting in $G$ vertex $a$ together with all edges $[v, b]$ with $v \in N(b)-N(a)$. Notice that $G^{\prime}$ is not an induced subgraph of $G$ and also that the simple neighborhood reduction corresponds to the case where $N(b)-$ $N(a)=\emptyset$.

\section{Minimizing posiforms}

Having observed the equivalence of the weighted maximum stable set problem and the maximization of a posiform, we may wonder whether the minimization of a posiform also has some interpretation. In fact, there is one which is illustrated by the following example:

$$
\text { Let } f=3 \bar{x}_{1} x_{2} \bar{x}_{3} x_{4}+4 \bar{x}_{1} \bar{x}_{3} x_{5}+\bar{x}_{4} \bar{x}_{5}+2 x_{2} \bar{x}_{5}
$$

We can associate with each term $T_{i}=\prod_{j \in A_{i}} x_{j} \prod_{k \in B_{i}} \bar{x}_{k}\left(\right.$ with $A_{i} \cap B_{i}=\emptyset$ ) a clause

$$
C_{i}=\left(\bigvee_{j \in A_{i}} \bar{x}_{j}\right) \bigvee\left(\bigvee_{k \in B_{i}} x_{k}\right)
$$

with weight $w(i)$.

Here we would have:

$$
\begin{array}{ll}
C_{1}=x_{1} \vee \bar{x}_{2} \vee x_{3} \vee \bar{x}_{4} & \text { with } w(1)=3 \\
C_{2}=x_{1} \vee x_{3} \vee \bar{x}_{5} & \text { with } w(2)=4 \\
C_{3}=x_{4} \vee x_{5} & \text { with } w(3)=1 \\
C_{4}=\bar{x}_{2} \vee x_{5} & \text { with } w(4)=2
\end{array}
$$


Assume we want to find an assignment of values true or false (1 or 0$)$ to the variables $x_{1}, \ldots, x_{4}$ such that the set of clauses that are satisfied has a maximum total weight.

Obviously, this is the so called weighted satisfiability problem (WSP)(Garey and Johnson, 1979). One sees that a clause $C_{i}$ will be violated if and only if the corresponding term $T_{i}$ is one.

So minimizing $f$ will minimize the total weight of the set of violated clauses; this amounts to maximizing the weight of the set of clauses that are satisfied. This is precisely the WSP.

Obviously to every WSP one can associate a posiform $f$ such that its minimum will solve the WSP. The two problems are thus equivalent.

Notice that the WSP may be interpreted as a weighted covering problem of edges by vertices in a hypergraph.

However a simple interpretation in terms of stable sets in a graph does not seem to be at hand for the minimization of a posiform.

\section{A brief conclusion}

We have seen here how some pseudo-Boolean methods may contribute to discovering purely graph theoretical operations that have proven to be essential in several procedures related to the stability number of graphs.

Besides this we have observed that minimizing a posiform is equivalent to the weighted MAX SAT problem, which is another famous integer programming problem.

This may again suggest new approaches and may lead to discovering other polynomially solvable cases as well.

It is likely that there are still many situations in graph theory and in combinatorial optimization in general where such pseudo-Boolean methods will suggest original ways of computing exact or approximate solutions.

\section{References}

Alexe, A., P.L. Hammer, V.V. Lozin, and D. de Werra. (2004). “Struction Revisited.” Discrete Applied Mathematics, 132, 27-46.

Berge, C. (1973). Graphs and Hypergraphs. North-Holland: Amsterdam.

Ebenegger, C., P.L. Hammer, and D. de Werra. (1984). "Pseudoboolean Functions and Stability of Graphs." Annals of Discrete Mathematics, 19, 83-98.

Hamor, A. (1980). "Stories of the One-Zero-One Nights: Abou Boul in Graphistan.” In P. Hansen and D. de Werra (Eds.), Regards sur la théorie des graphes. Lausanne, Presses Polytechniques Romandes, pp. 29-38.

Hertz, A. (1995). "Polynomially Solvable Cases for the Maximum Stable Set Problem." Discrete Applied Mathematics, 60, 195-210.

Hertz, A. (1985). "On the Use of Boolean Methods for the Computation of the Stability Number." Discrete Applied Mathematics, 76, 183-203.

Garey, M.R. and D.S. Johnson. (1979). Computers and Intractability: A Guide to the Theory of NPCompleteness. New York, Freeman.

Hammer, P.L., N.V.R. Mahadev, and D. de Werra. (1985). "Stability in CAN-Free Graphs.” J. Combin. Theory, B38, 23-30.

Hammer, P.L., N.V.R. Mahadev, and D. de Werra. (1985). "The Struction of a Graph: Applications to CN-Free Graphs." Combinatorica, 5, 141-147. 